\title{
Simulation of IEEE 802.16e Physical Layer
}

\author{
Shweta Bhambure ${ }^{1}$, Dr. A. D. Jadhav ${ }^{2}$, Prof. S. A. Shirsat ${ }^{3}$ \\ ${ }_{1,2,3}$ (Electronics and Telecommunication department, University Of Pune, India)
}

\begin{abstract}
Growth in technology has led to unprecedented demand for high speed Internet access. IEEE 802.16e (Mobile WiMAX) is a wireless communication standard with high data transfer rates and good performance. It not only is efficient as compared to its counterpart technologies today (Wi-Fi and $3 G$ ), but also lays the foundation for $4 G$ mobile communication. In $4 G$ wireless communication systems, bandwidth is a precious resource, and service providers are continuously met with the challenge of accommodating more users within a limited allocated bandwidth. To increase data rate of wireless medium with higher performance, Mobile WiMAX uses Orthogonal Frequency Division Multiple Access (OFDMA). This paper describes the simulation of the physical layer of IEEE 802.16e using Simulink in Matlab 7.0 (R2010a). The system performance is evaluated considering the Signal to noise ratio (SNR) and Bit error rate (BER) parameters.
\end{abstract}

Keywords: 802.16 , OFDMA, Mobile WiMAX.

\section{INTRODUCTION}

The world is passionately accepting the new wireless communication methods and services. The global demand for multimedia data services has grown at a remarkable pace which has led to the expansion of system capacity in terms of the number of subscribers supported, higher data rate and ubiquitous coverage with high mobility. Broadband wireless access (BWA) systems have evolved as the solution for the persistent demand of these multimedia services. The IEEE 802.16e air interface standard extends its fixed wireless access predecessor, IEEE 802.16-2004, to support mobile broadband wireless access systems. Mobile WiMAX (Worldwide Interoperability for Microwave Access), is a broadband wireless solution that enables wireless data over long distances in a variety of ways, from point-to-point links to full mobile cellular type access. It is a wireless digital communications system that is intended for wireless "metropolitan area networks". This technology can provide broadband wireless access (BWA) up to 30 miles $(50 \mathrm{~km})$ for fixed stations, and $3-10$ miles $(5-15 \mathrm{~km})$ for mobile stations upto $75 \mathrm{mph}$. With WiMAX, WiFi-like data rates are easily supported, but the issue of interference is reduced. WiMAX operates on both licensed and non-licensed frequencies, providing a regulated environment and viable economic model for wireless carriers. IEEE 802.16e can operate in both Line-Of-Sight (LOS) and Non-Line-Of-Sight (NLOS) environments. In NLOS, the PHY specification is extended to $2-11 \mathrm{GHz}$ frequency band which aims to fight with fading and multipath propagation. Mobile WiMAX physical layer is based on Scalable OFDMA technology to support variable channel bandwidth demand [1]-[5].

Mobile WiMAX, based on scalable Orthogonal Frequency Division Multiple Access (OFDMA) technology, is capable of simultaneously supporting fixed, portable, and mobile usage models. With OFDMA users share subcarriers and time slots. With scalable OFDMA, operators no longer need to choose between fixed or mobile services. The Mobile WiMAX Air Interface adopts Orthogonal Frequency Division Multiple Access (OFDMA) for improved multi-path performance in non-line-of-sight environments. Scalable OFDMA (SOFDMA) is introduced in the IEEE 802.16e Amendment to support scalable channel bandwidths from 1.25 $\mathrm{MHz}$ to $20 \mathrm{MHz}$. Also FFT size is variable ranging from 128, 512, $1024 \& 2048$. The scalability is supported by adjusting the FFT size while fixing the sub-carrier frequency spacing at $10.94 \mathrm{kHz}$. The variable FFT size allows for optimum operation of the system over a wide range of channel bandwidths and radio conditions. In OFDM (Orthogonal Frequency Division Multiplexing) the data is broken down into separate symbols and transmitted on different frequencies. OFDMA is same as OFDM except separate carriers assigned to separate users at the same time. OFDMA is a combination of OFDM and FDMA. It gives flexibility in channel assignment in time and frequency [5]-[9].

The modeling setup includes MATLAB 7.0 (R2010a), Simulink and Communications Block set running on Windows XP SP2/Windows 7. MATLAB 7.0 (R2010a) Simulink includes all the required function blocks as specified by the standard documents. 
This paper divides in VI sections. Section II gives related work. Section III explains the system block diagram of Physical layer of IEEE 802.16e. Matlab Simulink model for the IEEE 802.16e is presented in Section IV. Section IV also shows a few experimental results shown. Concluding remarks are given in section $\mathrm{V}$.

\section{LITERATURE REVIEW}

Author [1] has evaluated the performance of Mobile WiMAX for different modulation schemes. The following can be concluded: when channel conditions are poor, energy efficient schemes such as BPSK or QPSK were used and as the channel quality improves higher modulation schemes are used, making modulation schemes adaptive as per the channel conditions.

Author [2] describes the current trends in WiMAX systems for achieving high speed mobile wireless access services and outlined the technologies supporting these systems. The performance of the WiMAX-PHY layer based on the IEEE 802.16e standard was evaluated and assessed at different: (i) modulation schemes; (ii) coding rates.

Author [3] explains the OFDM block for Mobile WiMAX giving the details of OFDMA and Scalable OFDMA.

Author [4] demonstrates the theory related to the various adaptive modulation schemes of WiMAX.

Author [6] gives the performance analysis of Mobile WiMAX using scatter plots and BER and SNR graphs for different channel conditions.

\section{PhySICAL LAYER}

Physical layer set up the connection between the communicating devices and is responsible for transmitting the bit sequence. It also defines the type of modulation and demodulation as well as transmission power. WiMAX physical layer is based on the orthogonal frequency division multiple access (OFDMA) having frequency band below $11 \mathrm{GHz}$ and use TDD and FDD as its duplexing technology. OFDMA is a good choice of high speed data transmission, multimedia communication and digital video services. It even can maintain very fast data rate in a non line of sight condition and multipath environment. The role of the PHY-layer is to encode the binary digits that represent MAC frames into signals and to transmit and receive these signals across the communication media.

In Mobile WiMAX, the FFT size is scalable from 128 to 2,048. Here, when the available bandwidth increases, the FFT size is also increased such that the subcarrier spacing is always $10.94 \mathrm{kHz}$. This keeps the OFDM symbol duration, which is the basic resource unit, fixed and therefore makes scaling have minimal impact on higher layers. A scalable design also keeps the costs low. The subcarrier spacing of $10.94 \mathrm{kHz}$ can support vehicular mobility up to $125 \mathrm{kmph}$ when operating in $3.5 \mathrm{GHz}$. A subcarrier spacing of $10.94 \mathrm{kHz}$ implies that $128,512,1,024$, and 2,048 FFT are used when the channel bandwidth is $1.25 \mathrm{MHz}, 5 \mathrm{MHz}, 10 \mathrm{MHz}$, and $20 \mathrm{MHz}$, respectively. It should, however, be noted that mobile WiMAX may also include additional bandwidth profiles. This obviously will require different subcarrier spacing and hence will not have the same scalability properties.

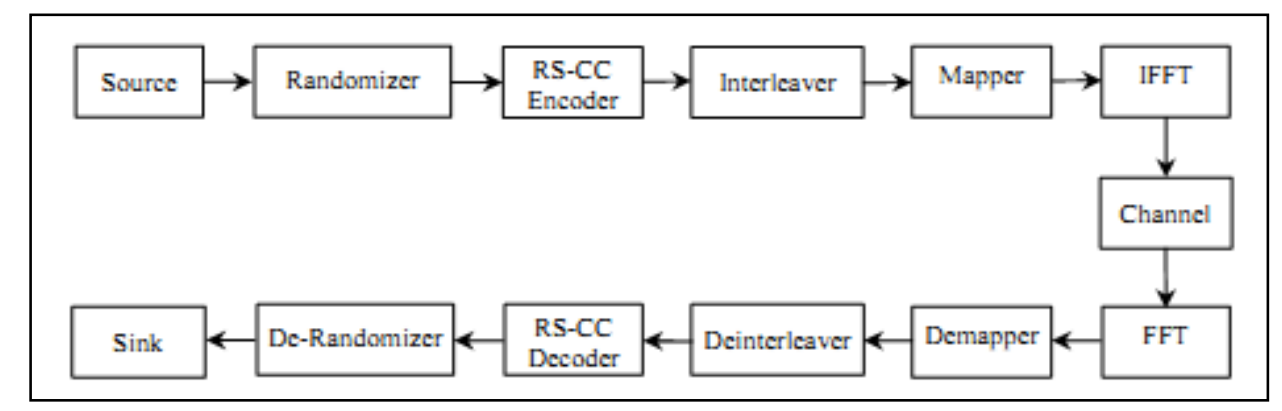

Fig 1 IEEE 802.16e Physical Layer [1]

The Physical layer has a transmitter, a receiver, and a channel over which the information is sent. The information signal is randomized to prevent a long sequence of $1 \mathrm{~s}$ and $0 \mathrm{~s}$. The randomized data bits are thereafter encoded using Reed Solomon (RS) encoder followed by Convolutional Coder (CC). The RS encoding 
is suitable for correction of burst type of error whereas the convolutional encoding is for random error. After RS-CC encoding all encoded data bits shall be interleaved by a block interleaver. In traditional block interleaver bits received from the encoder are stored row wise in the interleaver's memory. As soon as the memory is completely filled, the bits are read in a column-by-column manner. After interleaving, data passes through the mapper block in which modulation takes place. The resulting data symbols are used to construct one OFDM symbol by performing Inverse Fast Fourier Transform (IFFT). Cyclic Prefix (CP) is used to reduce Inter Symbol Interference (ISI) and Inter Carrier Interference (ICI). In the receiver, inverse blocks are applied which perform DFT, de-mapping, deinterleaving, decoding and de-randomizing operations in sequential manner to get back the original data bits.

The receiving chain is the mirroring for the transmitting chain blocks. In this work, the same blocks on the transmitting side were reversed and slightly modified with few blocks being replaced or modified significantly. The only replaced block is the Viterbi decoder that performs the reverse function of the convolutional encoder to correct any erroneous bits.

\section{SIMULINK MOdEL AND SIMULATION RESUlTS}

The Simulink model built is based on the following parameters and specifications. Table 1 defines the code rates for the different modulation schemes available. Table 2 shows a particular bandwidth-FFT pair specifications.

TABLE 1

MANDATORY CHANNEL CODING PER MODULATION [9]

\begin{tabular}{|l|c|c|c|l|l|}
\hline Modulation & $\begin{array}{l}\text { Uncoded block size } \\
\text { (bytes) }\end{array}$ & $\begin{array}{l}\text { Coded block size } \\
\text { (bytes) }\end{array}$ & $\begin{array}{l}\text { Overall } \\
\text { rate }\end{array}$ & RS- code & $\begin{array}{l}\text { CC code } \\
\text { Rate }\end{array}$ \\
\hline BPSK & 12 & 24 & $1 / 2$ & $(12,12,0)$ & $1 / 2$ \\
\hline 4 QAM & 24 & 48 & $1 / 2$ & $(32,24,4)$ & $2 / 3$ \\
\hline 4 QAM & 36 & 48 & $3 / 4$ & $(40,36,2)$ & $5 / 6$ \\
\hline 16-QAM & 48 & 96 & $1 / 2$ & $(64,48,8)$ & $2 / 3$ \\
\hline 16-QAM & 72 & 96 & $3 / 4$ & $(80,72,4)$ & $5 / 6$ \\
\hline 64-QAM & 96 & 144 & $2 / 3$ & $(108,96,6)$ & $3 / 4$ \\
\hline 64-QAM & 108 & 144 & $3 / 4$ & $(120,108,6)$ & $5 / 6$ \\
\hline
\end{tabular}

TABLE 2

PHYSICAL LAYER SPECIFICATIONS

\begin{tabular}{|l|l|}
\hline Bandwidth & $10 \mathrm{MHz}$ \\
\hline Sample Time & $89.3 \mathrm{~ns}$ \\
\hline FFT Size & 1024 \\
\hline Subcarrier Frequency Spacing & $10.94 \mathrm{kHz}$ \\
\hline Useful Symbol Time & $91.4 \mu \mathrm{s}$ \\
\hline Guard Time & $11.4 \mu \mathrm{s}$ \\
\hline OFDMA Symbol Time & $102.8 \mu \mathrm{s}$ \\
\hline
\end{tabular}

Fig 2 shows the Simulink model for IEEE 802.16e. The model built has RS code rate $3 / 4$ and CC code rate $5 / 6$ with carrier frequency below $11 \mathrm{GHz}$. The model consists of three main components namely transmitter, receiver and channel. The transmitter and receiver components are as explained in the above sections, and the channel is modeled as AWGN. The only replaced block is the Viterbi decoder that performs the reverse function of the convolutional encoder to correct any erroneous bits. 


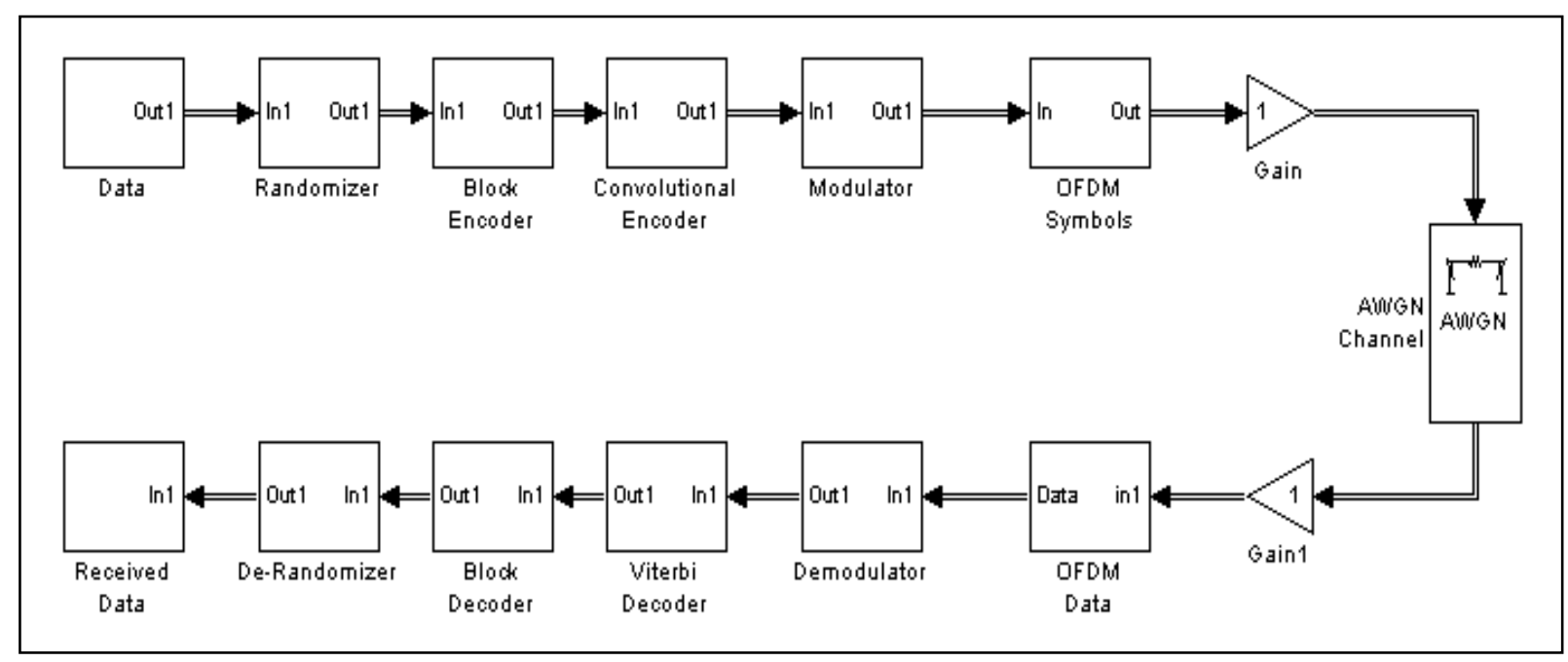

Fig 2 Simulink modelof IEEE 802.16e

The performance evaluation of the system is done using the parameters Signal to noise ratio (SNR) and Bit error rate (BER). Based on the model presented in this paper, and tests carried out, the performance is displayed in the following figure in terms of the BER versus SNR logarithmic plot, time-scatter plots.

\section{A. BIT ERROR RATE}

The number of error bits occurring within the transmitted signal is called Bit Error Rate (BER). We can define BER as follows,

\section{B. $E_{b} / N_{0}$}

$$
B E R=\frac{\text { Error } \text { Number }}{\text { Total number of bits sent }}
$$

Energy per bit to noise power spectral density ratio plays an important role in communication system. The normalized form of $\mathrm{E}_{\mathrm{b}} / \mathrm{N}_{0}$ is Signal-to- Noise Ratio (SNR).

C. BER vs $E_{b} / N_{0}$

The Bit Error Rate (BER) defined as the probability of error (Pe), on the other hand Signal-to- Noise is the term of power ratio between a signal and background noise. The probability of error is proportional to $E_{b} / N_{0}$

In Fig 3 we can see the transmitted and recovered information signals obtained on a scope. Fig. 4 shows the scatter plot of 4-QAM. The scatter plot obtained on a discrete time scatter plot scope block displays scatter plots of a modulated signal, to reveal the modulation characteristics, such as channel distortions of the signal.

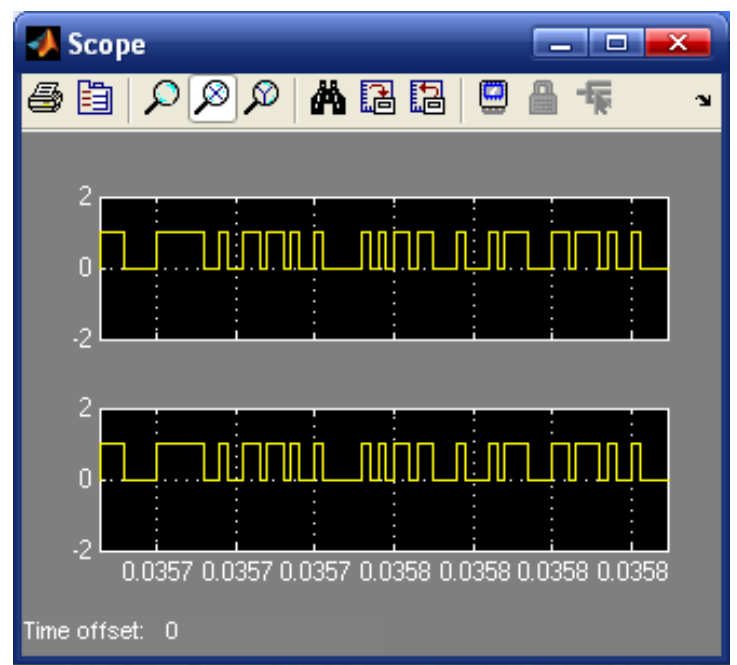

Fig 3 Transmitted and Recovered Information Signals 


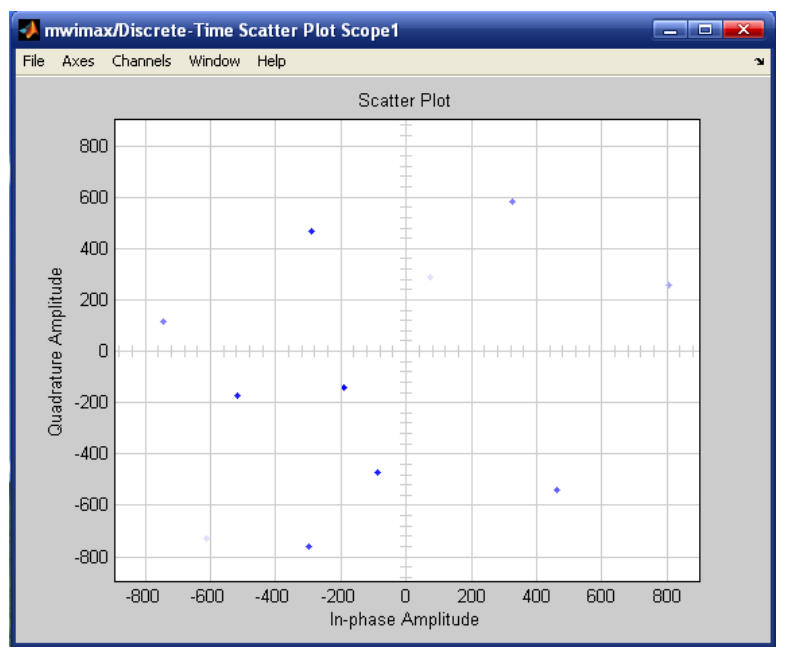

Fig 4 Scatter Plot for 4-QAM

In Fig. 5 we see the BER vs. $\mathrm{E}_{\mathrm{b}} / \mathrm{N}_{0}$ graphs for the various modulation schemes, namely 4-QAM, $16-$ QAM and 64-QAM for AWGN channel. Simulation results show that 4 QAM has good BER vs. Eb/No performance than 16 QAM and 64 QAM. Thus spectrally efficient modulation schemes have poor BER vs. SNR performance.

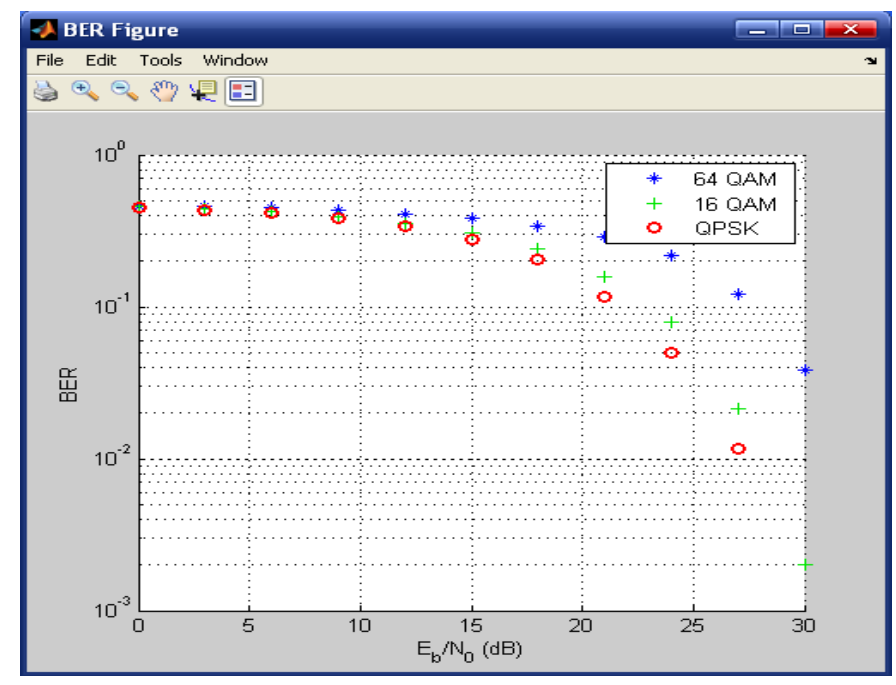

Fig 5 BER vs. $\mathrm{E}_{\mathrm{b}} / \mathrm{N}_{0}$ Graphs for different modulation schemes

\section{CONCLUSION}

Simulation was the methodology used to investigate the Physical layer performance. The performance of the developed model is studied using parameters like BER, SNR and scatter plots. The Simulink model developed is studied for the various modulation schemes (QPSK, 16 QAM and 64 QAM). In future this model can be expanded to include the components of the MAC layer and a complete end to end WiMAX system could be built based on this model. Also scalability can be implemented.

\section{Acknowledgements}

I would like to take this opportunity to express my sincere appreciation to my guide, Dr. A. D. Jadhav for his guidance and assistance, and for the help he provided throughout this Master program and for the continuous encouragement and support.

And finally thanks to all my friends. Thanks to all the people who helped me by any means. 


\section{REFERENCES}

[1] M. A. Hasan, "Performance Evaluation of WiMAX/IEEE 802.16 OFDM Physical Layer," Elsinki University of Technology, espoo, pp.1-33, June 2007.

[2] M.A. Mohamed, F.W. Zaki1, R.H. Mosbeh / Simulation of WiMAX Physical Layer: IEEE 802.16e / International Journal of Computer Science and Network Security, November 2010.

[3] U. S. Jha and R. Prasad, OFDM towards Fixed and Mobile Broadband Wireless Access, Artech House Publisher, London, 2007.

[4] B. Chaitanya, T.S. Prasad, K. Sruthi, T. Tejaswi, "Adaptive Modulation Techniques for WIMAX," (IJCNS) International Journal of Computer and Network Security, Vol. 2, No. 5, May 2010.

[5] The WiMAX Forum. www.wimaxforum.org

[6] Gazi Faisal Ahmed Jubair, Muhammad Imran Hasan, Md. Obaid Ullah / Performance Evaluation of IEEE 802.16e (Mobile WiMAX) in OFDM Physical Layer / Blekinge Institute of Technology, August 2009

[7] A. Ghosh J. Andrews and R. Muhamed, Fundamentals of WiMAX: Under- standing Broadband wireless Networking, Pearson Education, Inc., 2007.

[8] IEEE, IEEE Standard for Local and metropolitan area networks, Part16: Air Interface for Fixed and Mobile Broadband Wireless Access Systems, 2006.

[9] ETSI, "www.etsi.org" 